\title{
PAH anions as carriers of the mid-IR emission bands in planetary nebulae
}

\author{
Ryszard Szczerba ${ }^{1}$, Christine Joblin ${ }^{2}$, Olivier Berné ${ }^{2}$, and \\ Cezary Szyszka ${ }^{3}$ \\ ${ }^{1}$ N. Copernicus Astronomical Center, \\ Rabiańska 8, 87-100 Toruń, Poland \\ email: szczerba@ncac.torun.pl \\ ${ }^{2}$ Centre d'Etude Spatiale des Rayonnements, Université Toulouse 3 et CNRS, \\ 9 Av. du Colonel Roche, 31028 Toulouse cedex 04, France \\ ${ }^{3}$ Nicolaus Copernicus University, \\ Gagarina 11, 87-100 Toruń, Poland
}

\begin{abstract}
We present results of the mid-IR spectra decomposition for planetary nebulae and compact H II regions in our Galaxy and Magellanic Clouds. The striking correlation between the required PAH component with "7.7" $\mu \mathrm{m}$ band shifted to about $7.8 \mu \mathrm{m}$ and electron densities of the modeled sources allows us to argue that this PAH component may be in fact PAH anions $\left(\mathrm{PAH}^{-}\right)$.
\end{abstract}

Keywords. (ISM:) dust, extinction, (ISM:) planetary nebulae: general, infrared: ISM

\section{Introduction}

Among the different dust populations, the carriers of the so-called aromatic infrared bands (AIBs) are the subject of a long debate. The main AIBs are located at 3.3, 6.2, $7.7,8.6,11.3,12.7 \mu \mathrm{m}$ and are attributed to very small aromatic dust particles amongst which there are large polycyclic aromatic hydrocarbons (PAHs). However, these band positions are varying. In particular, the dominant band in the "7.7" $\mu \mathrm{m}$ complex arises at $7.8 \mu \mathrm{m}$ in planetary nebulae (PNe) compared to $7.6 \mu \mathrm{m}$ in compact-H II (c-H II) regions (Peeters et al. 2002) or in photo dissociation regions (PDRs). These shifts in the AIB positions may be related to chemical or physical changes of the carriers in different environments. To investigate possible evolution of the AIB carriers, Joblin et al. (2008) have analyzed spectra of several PNe and c-H II regions in the Milky Way (MW) and in the Large and Small Magellanic Clouds (LMC \& SMC). Their analysis is based on the results of Berné et al. (2007) and Rapacioli et al. (2005) and uses a decomposition of the mid-IR spectra into a set of template spectra (see Sect.2).

In this paper we discuss results of the above analysis for selected $\mathrm{PNe}$ and $\mathrm{c}-\mathrm{H}$ II regions in MW and LMC and show that the obtained decomposition may be understood by the presence of different amount of $\mathrm{PAH}$ anions in environments with different ionization parameter $\left(U_{\text {ion }}\right)$.

\section{Templates and the spectra decomposition}

Rapacioli et al. (2005) and Berné et al. (2007) were able to extract the spectra of PAH neutrals $\left(\mathrm{PAH}^{0}\right), \mathrm{PAH}$ cations $\left(\mathrm{PAH}^{+}\right)$and very small grains (VSGs) from the mid-IR spectra of the analyzed PDRs. Joblin et al. (2008) have built three template spectra 
Table 1. Relative amount of $\mathrm{PAHs}$ in $[\%]\left(\mathrm{PAH}^{0}+\mathrm{PAH}^{+}+\mathrm{PAH}^{\mathrm{x}}=100 \%\right)$ required for the fits presented in Figure 1.

\begin{tabular}{|c|c|c|c|c|c|c|c|c|}
\hline Object & \multicolumn{8}{|c|}{$\left|\mathbf{P A H}^{0}[\%]\right| \mathbf{P A H}^{+}[\%]\left|\mathbf{P A H ^ { x }}[\%]\right| \mathbf{N}_{\mathrm{el}}\left[\mathrm{cm}^{-3}\right] \mid \operatorname{Ref}$} \\
\hline Vo 1 & 4 & | & 0 & | & 96 & | & $10^{5}$ & 1: \\
\hline SMP LMC 99 & 69 & | & 0 & | & 31 & | & 3600 & | 2: \\
\hline IRAS $22308+5812$ & 48 & | & 39 & | & 13 & | & 770 & 3: \\
\hline MSX LMC 1798 & 60 & | & 40 & | & 0 & | & $?$ & | \\
\hline
\end{tabular}

Ref - 1: Menzies \& Wolstencroft (1990); 2: Leisy \& Dennefeld (2006); 3: Martín-Hernández et al. (2002)

from these results. However, the basis made of the three PDR components was found to be insufficient to obtain a satisfactory fit to the observations of PNe. Broad emission features around 8 and $12 \mu \mathrm{m}$, which are known to be prominent in post-Asymptotic Giant Branch (post-AGB) star spectra (see, e.g., Kwok et al. 2001), appear to be still present in the spectra of $\mathrm{PNe}$. As template for these features we chose a simplified gaussian profiles which provide a reasonable fit to the bands observed in the post-AGB star IRAS 13416-6243, a prototype of C-type object from Peeters et al. (2002). In addition, the "7.7" $\mu \mathrm{m}$ band is shifted to near $7.8 \mu \mathrm{m}$ in PNe and it was necessary to introduce an additional $\mathrm{PAH}$-type (hereafter $\mathrm{PAH}^{\mathrm{x}}$ ) template with the peak position shifted to the red. Altogether, we have used a set of six template spectra to perform decomposition of the mid-IR spectra of PNe and c-H II regions (see Joblin et al. 2008 for details).

The obtained decompositions for two PNe (Vo 1 from MW and SMP LMC 99 from LMC) and two c-H II regions (IRAS 22308+5812 from MW and MSX LMC 1798 from LMC) are shown in Figure 1. Only PAH components are displayed (see figure caption). In Table 1 the relative amount of the different PAH components required for the fits is collected. A striking correlation is found between the required amount of $\mathrm{PAH}^{\mathrm{x}}$ and electron densities $\left(N_{e l}\right)$ of the modeled sources. Joblin et al. (2008) have discussed the possibility that $\mathrm{PAH}^{\mathrm{x}}$ may be in fact $\mathrm{PAH}$ anions, on the basis of trends in bands shift observed in theoretical data from Malloci et al. (2007). To further progress on this suggestion we have computed the charge of $\mathrm{PAH}$ molecules in environments characterized by a different ionization parameter $\left(U_{\text {ion }}=Q_{H} /\left(4 \pi R_{S}^{2} N_{e l} c\right)\right.$; where $Q_{H}$ is the number of H-ionizing photons, $R_{S}$ is the Strömgren radius and c is the light speed).

\section{PAH charge}

The results of the PAH charge computations are shown in Figure 3. The method used to compute the PAH charge is the same as described in Stasińska \& Szczerba (2001). However, the physics of the dust-gas-radiation interaction was updated according to Weingartner \& Draine (2001) and the neutral PAH properties from Li \& Draine (2001) were implemented. Our method used to compute the charge is applicable to large dust particles, where the dust charge may be treated as a continuous quantity and is probably not correct for so small dust particles as PAHs. However, we have checked that the computed PAH charge when rounded to the greatest integer charge less than the computed one (for instance -2 instead of -1.3 ) was never smaller than the most negative allowed charge (Eq. 24 of Weingartner \& Draine 2001). In addition, our method reproduces well the known result that dust charge is approaching $-2.16 \mathrm{eV}$ for low $U_{\text {ion }}$ (see PAH charge for $U_{\text {ion }}=1 . e-5$ in Figure 3 ). Therefore, we think that our results give a representative value of $\mathrm{PAH}$ charge in different environments. 


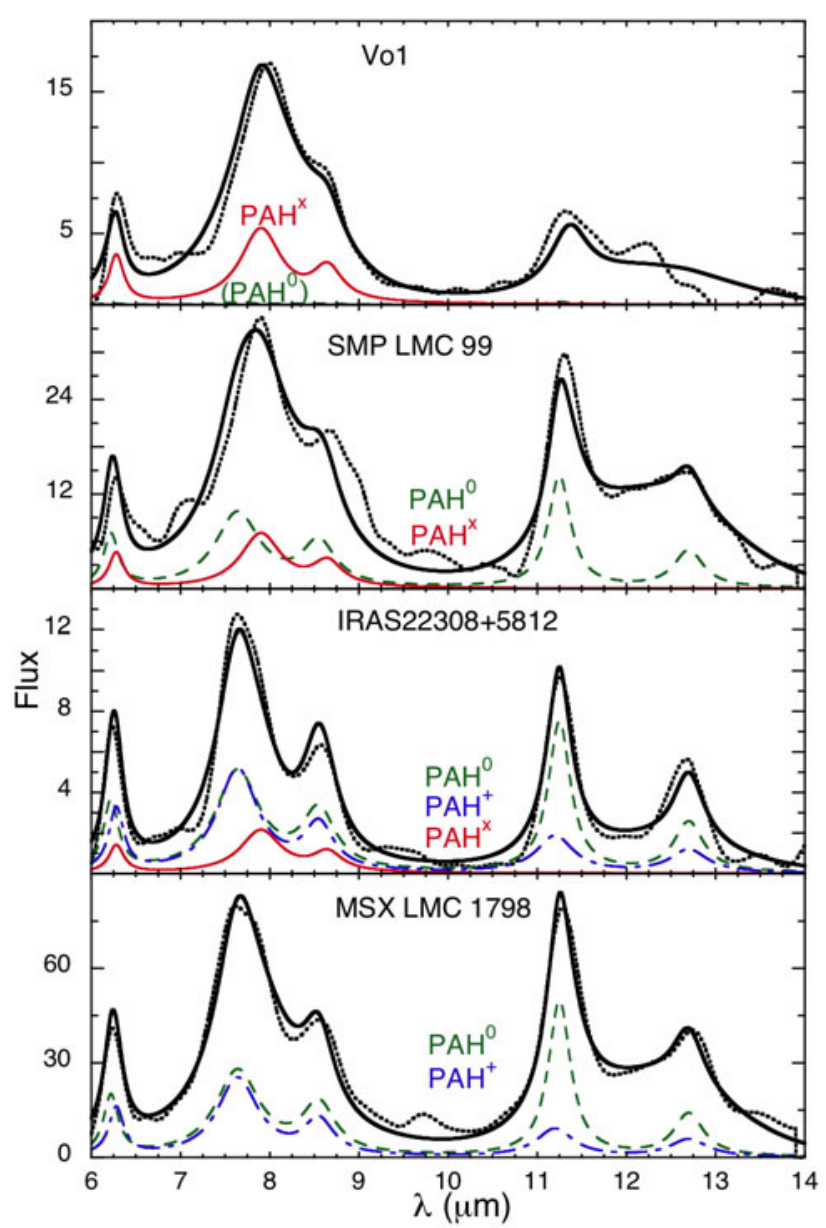

Figure 1. Mid-IR spectra after continuum subtraction and smoothing to the resolution of $\lambda / \Delta \lambda=45$ (dotted line) and fit obtained using a set of six template spectra (solid line). Below each fit, only $\mathrm{PAH}$ components are displayed: $\mathrm{PAH}^{0}$ (dashed line), $\mathrm{PAH}^{+}$(dash-dot line) and $\mathrm{PAH}^{\mathrm{x}}$ (thin solid line). On each panel, PAH components are listed (from top to bottom) according to their contribution to the fit.

At least three of the four selected objects (the two PNe and IRAS 22308+5812) show (roughly) similar $Q_{H}$ (they have similar effective temperatures and - roughly - luminosities) and $R_{S}$. The only striking difference between the parameters characterizing these objects, which enter into the ionization parameter, is the electron density (see Table 1). We may expect that the object with the highest electron density (Vo 1) will have the smallest ionization parameter and therefore a lot of PAH anions, while IRAS $22308+5812$ which has the lowest $N_{e l}$ will have the largest $U_{\text {ion }}$ and therefore the lowest amount of $\mathrm{PAH}^{-}$. For LMC c-H II region (MSX LMC 1798) we do not know $N_{e l}$, however, in general, the density of $\mathrm{H}$ II regions in galaxies with lower metallicity will be lower so their ionization parameters will be larger and therefore the percentage of $\mathrm{PAH}^{-}$may be negligible. Note that Cox \& Spaans (2006) have shown that the fraction of PAH anions is much lower in low metallicity environments, so our results are in line with their findings. 


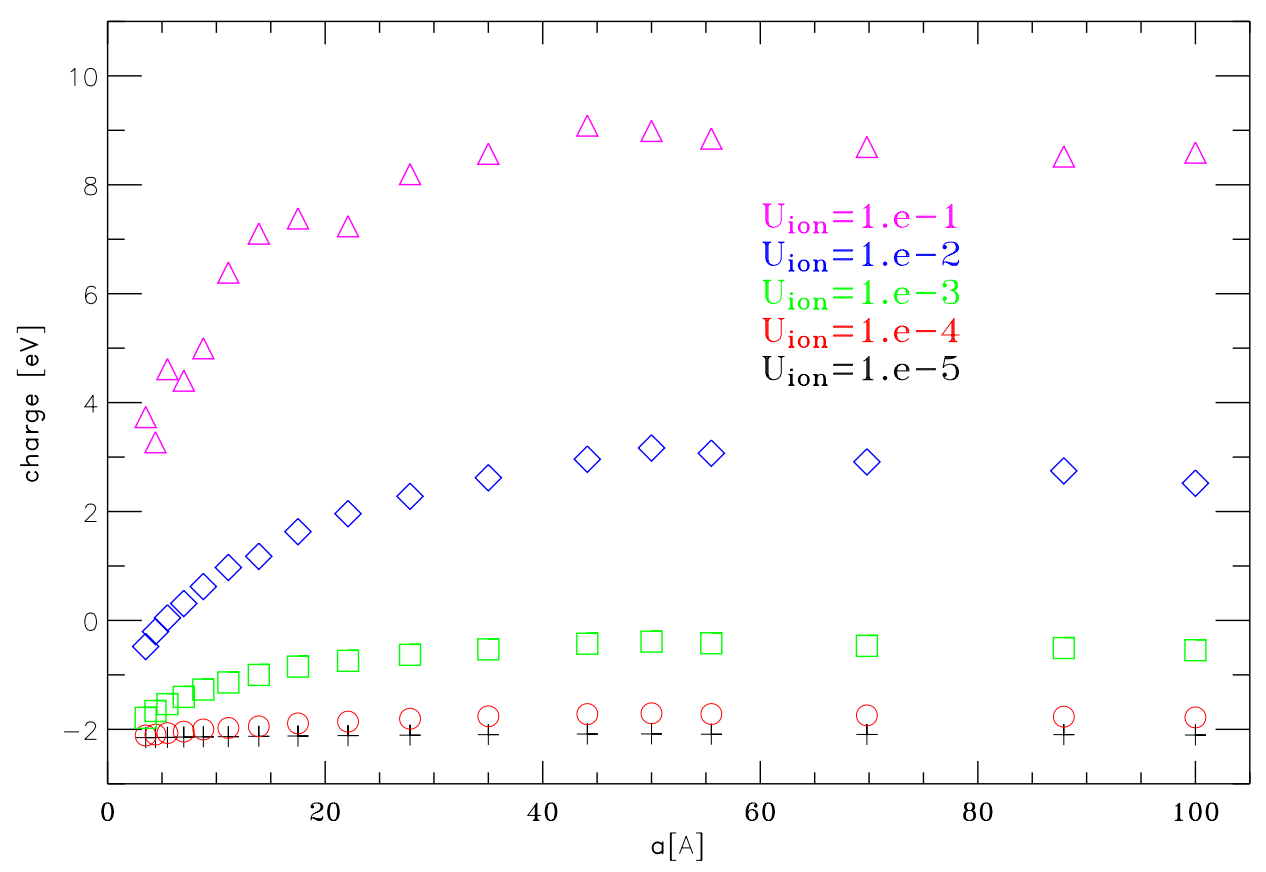

Figure 2. Calculated charge of PAH molecules with different size in environments characterized by a different ionization parameter $U_{\text {ion }}$ (pluses - 1.e-5; circles - 1.e-4, squares - 1.e-3, diamonds - 1.e-2; triangles - 1.e-1.) and a gas electron temperature of $10^{4} \mathrm{~K}$.

\section{Acknowledgements}

R.Sz. acknowledges support from grant N203 019 31/2874 of the Science and High Education Ministry of Poland. Support from the French national program, Physique et Chimie du Milieu Interstellaire, is also acknowledged.

\section{References}

Berné, O., Joblin, C., Deville, Y., Smith, J. D., Rapacioli, M., Bernard, J. P., Thomas, J., Reach, W., \& Abergel, A. 2007, $A \& A, 469,575$

Cox, N. \& Spaans, M. 2006, A\&A, 451, 973

Joblin, C., Szczerba, R., Berné, O., \& Szyszka, C. 2008, A\&A submitted

Kwok, S., Volk, K., \& Bernath, P. 2001, ApJ (Letter), 554, L87

Leisy, P. \& Dennefeld, M. 2006, A\&SA, 456, 451

Li, A., \& Draine, B. T. 2001, ApJ, 554, 778

Malloci, G., Joblin, C., \& Mulas, G. 2007, Chem. Phys., 332, 353

Martín-Hernández, N. L., Peeters, E., Morisset, C., Tielens, A. G. G. M., Cox, P., Roelfsema, P. R., Baluteau, J.-P., Schaerer, D., Mathis, J. S., Damour, F., Churchwell, E., Kessler, M. F. 2002, $A \& A, 381,606$

Menzies, J. W. \& Wolstencroft, R. D. 1990, MNRAS, 247, 177

Peeters, E., Hony, S., Van Kerckhoven, C., Tielens, A. G. G. M., Allamandola,L.J., Hudgins, D. M., \& Bauschlicher, C. W. 2002, A\& A, 390, 1089

Rapacioli, M., Joblin, C., \& Boissel, P. 2005, A\& A, 429, 193

Stasińska, G. \& Szczerba, R. 2001, A\&A, 379, 1024

Weingartner, J. C. \& Draine, B. 2001, ApJS, 134, 263 


\section{Discussion}

SARRE: I would like to ask what molecular form PAH anions have? Are they stable closed shell systems, small example would be coronene with electrons attached, or are they radicals with electrons added to make them closed shell of anions?

SzCZERBA: It is difficult to make a definite statement. However, the spectral agreement is based on the theoretical spectral database of PAHs http://astrochemistry.ca.astro.it/ database/ in which most of the PAH species considered are closed shell as neutrals and open shell as singly ionized (cations or anions).

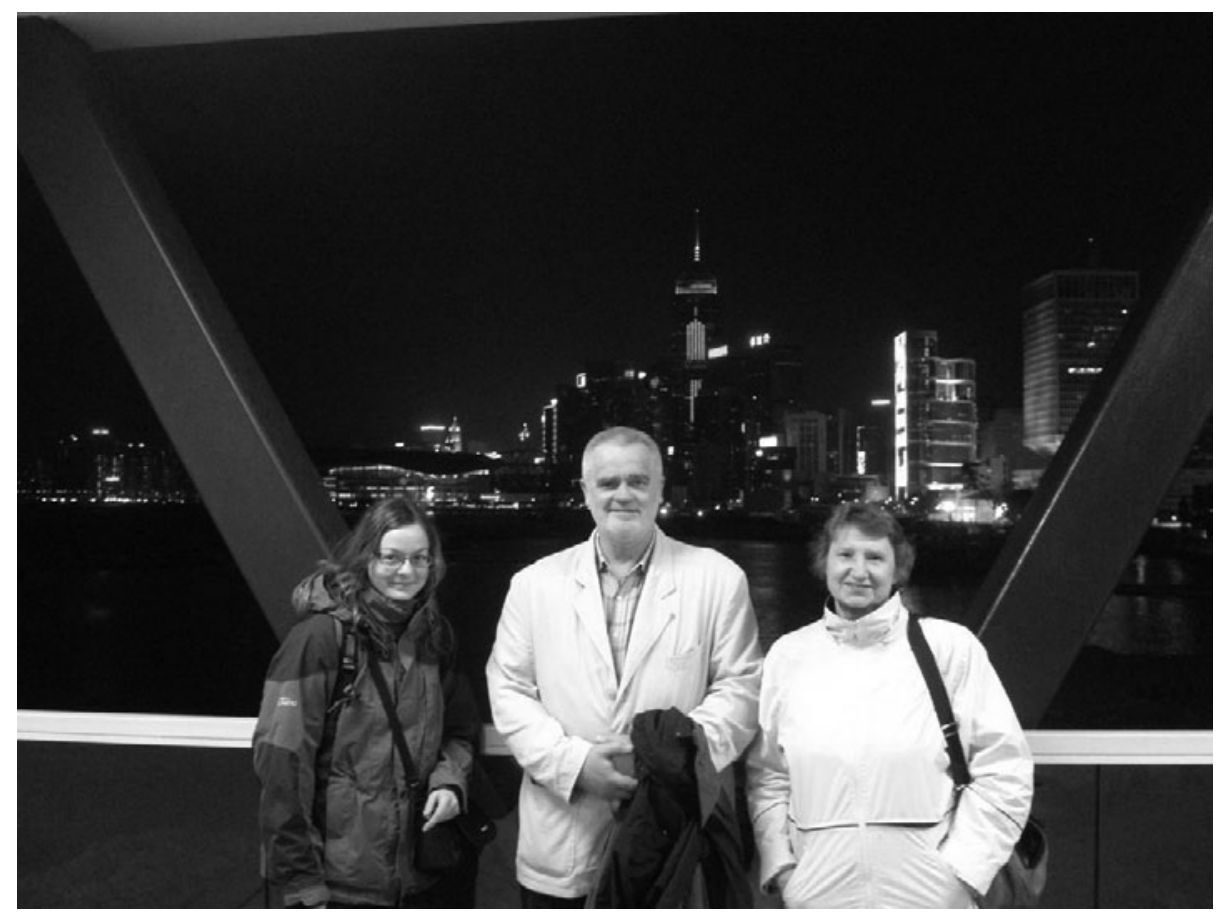

Maja Kazmierczak, Ryszard Szczerba, and Vera K. Rosenbush in front of the Wan Chai habour (photo by Yuriy Serozhkin). 


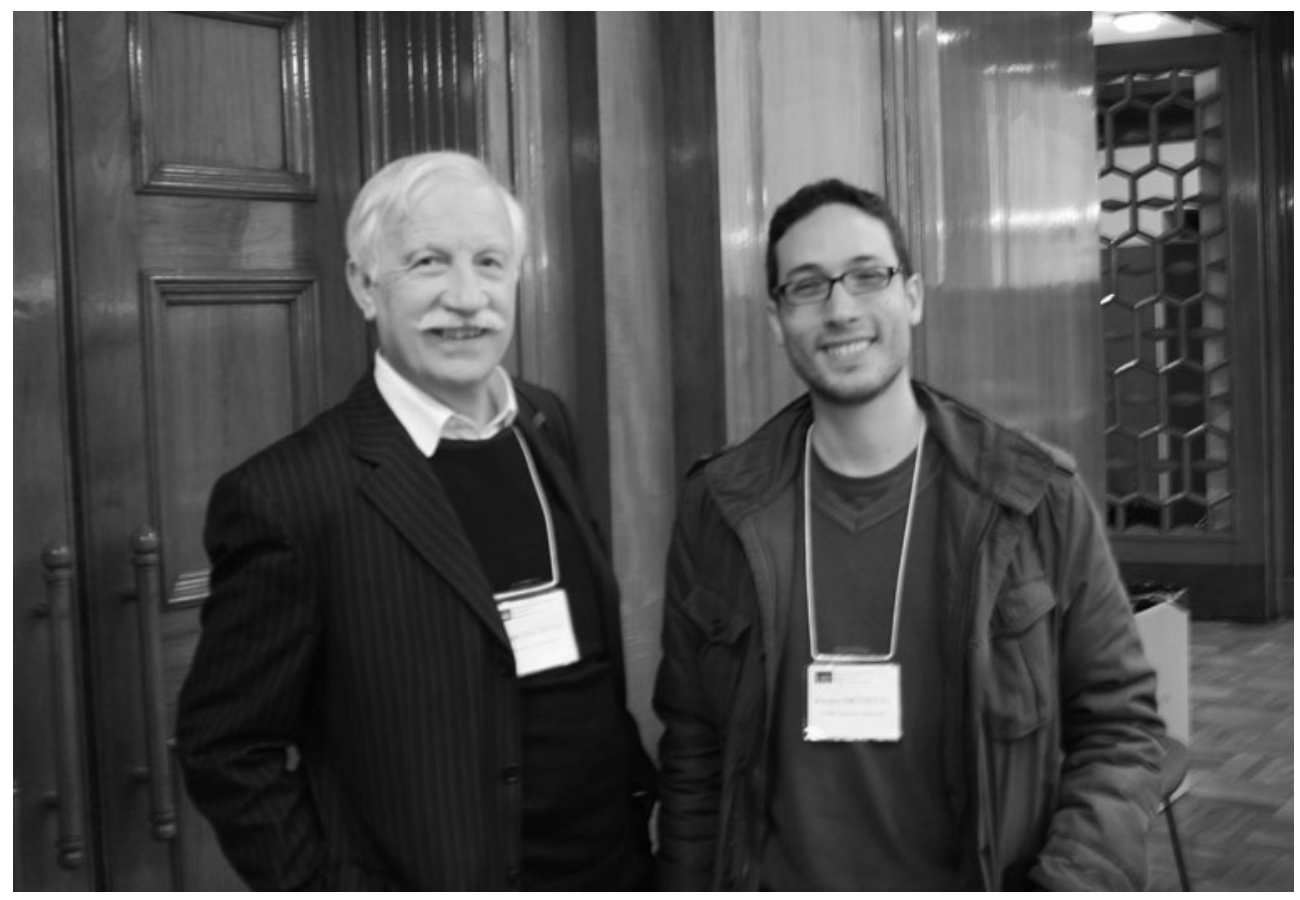

Philippe Bréchignac and Rosario Brunettto. 